\title{
On the computation of option prices and Greeks under the CEV model
}

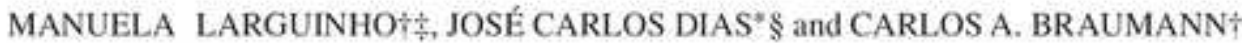 \\ fCentro de Investigaçẵo em Matemátiea e Aplicaçōes, Universidade de Évora, Rua Romāo Ramalho 59. \\ $7000-671$ Evora, Portugal \\ stSCAC, Quinta Agricola. Bencanta, 3040-316 Coimbra, Portugal \\ \$BRU-UNIDE and ISCTE-IUL Business School, Complexo INDEG/ISCTE, Av. Prof. Anibal Bettencourt. \\ I600-189 Lisboa, Portugal
}

(Received 2.May 2011; in final form 4 Jamuary 2013)

\begin{abstract}
Pricing omions and evaluating Grecks under the constant elasticity of variance (CEV) model requires the computation of the non-central chi-square distribution function. In this article, we compare the performance, in terms of accuracy and computational time, of altemative methods for cointruting such probatility distributions against an extemally tested benchuark. In addition, we present closed-form soletions for computing Greek measures under the unrestricted CEV opkion pricing model, thas being able to accommoodate difect leverage effocts as well as invere leverage effects that are frequently
\end{abstract} observed in options markets.

Keywords: Option priciag: Derivatives hedging: Computational finance: Statistical methods

JEL. Classificunion: G1. G13

\section{Introduction}

Every option pricing model has to make a key assumption regarding the 'right' distribution to be used when discounting the eption's expected payoff. This expectation is typically computed by integrating the payoff function over a riskneutral density function. Under the log-normal models of Black and Scholes (1973) and Mertion (1973) (the BSM model) it is assumed that the underlying asset price follows : geometric Brownian motion. Yet, this prediction has been convincingly rejected in the firance literature. For instance, it is well documented-sec, forexample, Jackwerth and Rubinstein (1996) - that the log-nomal assumption is unable to accommodate the negative skewness and the high kurtosis that are usually implicit in empirieal asset return distributions.

The constant elasticity of variance (CEV) model of Cox (1975) is consistent with two well-known facts that have found empirical support in the fiterature: the existence of a negative correlation between stock retums and realized volatility (leverage effect), as observed, for instance, by Bekaert and Wu $(2000)$, and the inverse relation between the implied volatility and the strike price of an option contract (implied volatility skew) - \$ee, for example, Dennis and Mayhew (2002). More importantly, being a 'local volatility' model, the CEV diffusion

"Corresponding authot. Enail: jose carfos, dias (itiscte.pt is consistent with a 'complete market' setup and, therefore, allows the hedging of short option positions only through the underlying asset.

Computing option prices under the CEV model typically involves the use of the so-called complementary non-central chi-square distribution function. There exists an extensive literature devoted to the efficient computation of this distribu. tion function, with several alternative representations available (see, for instance, Farebrother (1987), Posten (1989). Schroder (1989), Ding (1992), Kriesel and Bablok (1996). Benton and Krishnamoorthy (2003), and Dyrting (2004)). The complementary non-central chi-square distribution function ean also be computed using a method based on series of incomplete gamma furctions. For certain ranges of parameter values, some of the alternative representations available are more computationally efficient than the series of incomplete gamma functions. Moreover, for some parameter configurafions the use of analytic approximations (e.g. Sankaran (1963). Fraser et al. (1998), and Penev and Raykov (2000)) may be preferable.

The main purpose of this article is to provide comparative results in terms of accuracy and computation time for existing alternative algorithens for computing the non-central chisquare distribution function to he used for option pricing and hedging under the CEV model for a large set of parameter values. $A$ similarstudy has been conducted by $A g c a$ and $C$ hance 